\title{
Mach Number Effect on Flowfield over a Delta Wing in Supersonic Region
}

\author{
Akira Oyama $^{1}$ \\ JAXA Institute of Space and Astronautical Science, Sagamihara, Kanagawa, 229-8510, Japan \\ Masato Ito $^{2}$ \\ Aoyama Gakuin University, Sagamihara, Kanagawa, 229-0666, Japan, Currently, YAMAHA CORPORATION, \\ Japan \\ Genta Imai $^{3}$ \\ University of Tokyo, Sagamihara, Kanagawa,229-8510, Japan, currently, Mitsubishi Heavy Industries, LTD., \\ Japan \\ Seiji Tsutsumi ${ }^{4}$ \\ JAXA JEDI Center, Sagamihara, Kanagawa, 229-8510, Japan \\ Nobuo Amitani ${ }^{5}$ \\ Kogakuin University, Shinjuku, Tokyo, 192-0015, Japan \\ and \\ Kozo Fujii ${ }^{6}$ \\ JAXA Institute of Space and Astronautical Science, Sagamihara, Kanagawa, 229-8510, Japan
}

\begin{abstract}
To understand Mach number effect on flow field over a delta wing with blunt leading edge in supersonic and high angle of attack region, wind tunnel experiments of a $65^{\circ}$ delta wing are performed in supersonic and high angle of attack flow conditions at the JAXA's transonic / supersonic wind tunnel. Oil flow for surface flow visualization, pressure sensitive paint for surface pressure distribution measurement, and Schlieren images for shock wave visualization are used. The present results indicate that a delta wing with blunt leading edge can be mixed flow of two different types of flow structure in supersonic and high angle of attack flow region and the location of the boundary of the two types of flow moves toward the apex of the wing as the free-stream Mach number increases.
\end{abstract}

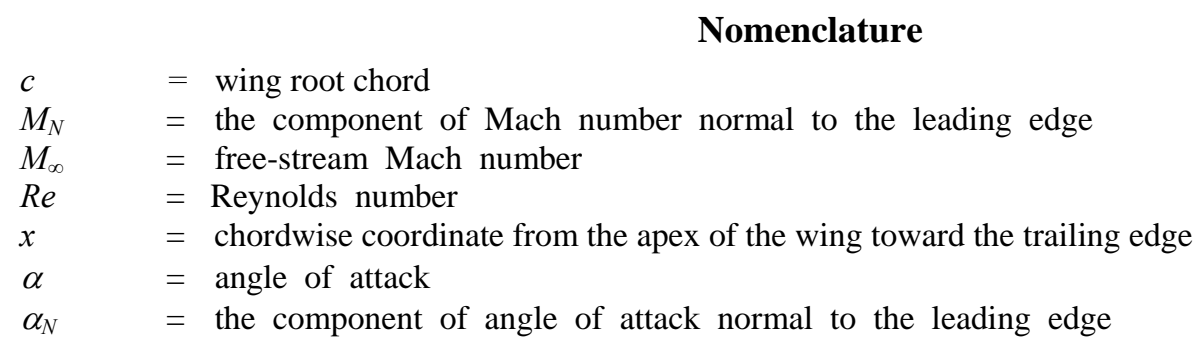

\footnotetext{
${ }^{1}$ Assistant Professor, Department of Space Transportation Engineering, 3-1-1 Yoshinodai, Member AIAA.

${ }^{2}$ Graduate Student, Department of Science and Engineering, 5-1-1 Fuchinobe, Student Member AIAA.

${ }^{3}$ Graduate Student, Department of Aeronautics and Astronautics, 3-1-1 Yoshinodai, Non-member.

${ }^{4}$ Researcher, Team for Utilization of Simulation Technology, 3-1-1 Yoshinodai, Member AIAA.

${ }^{5}$ Graduate Student, Major of Mechanical Engineering, 2665-1 Nakanomachi, Non-member.

${ }^{6}$ Professor, Department of Space Transportation Engineering, 3-1-1 Yoshinodai, Fellow AIAA.
} 


\section{Introduction}

$\mathrm{D}$ ELTA wing at high angle of in transonic or complex flowfield involving flow separation and shock wave generation. The earliest attempt to understand transonic and supersonic flows over delta wings at high angle of attack appeared in 1964 in the work of Stanbrook and Squire ${ }^{1}$. By examining all the experimental data available, they proposed a classification of the flow patterns based on the component of angle of attack normal to the leading edge and the component of Mach number normal to the leading edge (Fig. 1(a)). They classified the flows into two types; attached flow and separated flow at the leading edge.

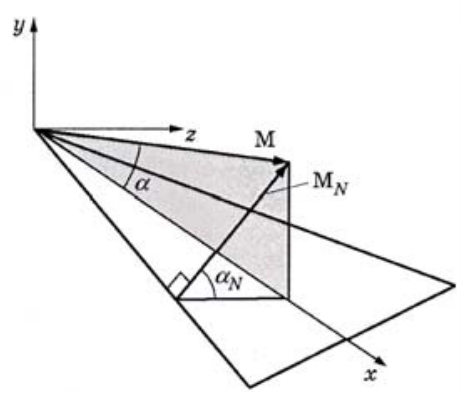

(a) Definition of $M_{N}$ and $\alpha_{N}$

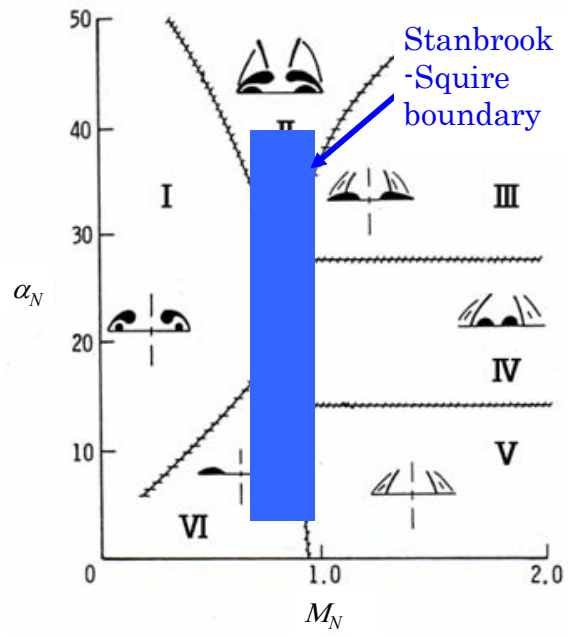

(b) Classification of flow field ${ }^{1,2}$
Figure 1. Classification of flow field of delta wing.

The boundary line between these two types existing near $M_{N}=1.0$ has come to be known as the Stanbrook-Squire boundary (Fig. 1(b)). Two decades later, Miller and Wood $^{2}$ experimentally studied flows over delta wings with different leading edge sweep angles using oil flow, tufts, and vapor screen methods. They classified the flows into six patterns according to $\alpha_{N}$ and $M_{N}$, namely, (I) Classical vortex, (II) Vortex with shock, (III) Separation bubble with shock, (IV) Shock-induced separation, (V) Shock with no separation and (VI) Separation bubble with no shock (Fig. 1(b)). Szodruch and Peake ${ }^{3}$ suggested a similar classification for much thicker wings than those used by Miller and Wood. Seshadri and Narayan ${ }^{4}$ and Brodetsky ${ }^{5}$ proposed similar classifications by examining flow fields in more detail. Recently, Imai, Fujii, and Oyama ${ }^{6}$ examined the flow mechanism determining the flow type by conducting computations of flow field over a 65-degrees sweep delta wing at high angles of attack in transonic and supersonic regions for better understanding of the flow mechanism behind the flow type classification of delta wing.

However, most of previous researches on delta wing focused on wing with sharp leading edge while practical delta wing has blunt leading edge. Delta wing with blunt leading edge generates more complex flow than that with sharp leading edge. For example, in subsonic flow region, delta wing with sharp leading edge produces its primary vortex at the apex of the wing while the primary vortex does not necessarily separates at the apex of the wing for delta wing with blunt leading edge. As a result, flow type of a delta wing with blunt leading edge becomes mixed flow of two different flow types.

Luckring $^{7-9}$ obtained extensive experimental data set to identify Reynolds number and Mach number (compressibility) effects on flow field over a $65^{\circ}$ delta wing with blunt leading edge. He showed that separation point of the primary vortex moves according to many flow and geometry properties such as leading-edge bluntness, angle of attack, Mach number, Reynolds number, and so on. However, his research is limited to subsonic and transonic flow regions. Though Seshadri and Narayan ${ }^{4}$ pointed out that mixed flow appears for a delta wing with sharp leading edge in high Mach number and high angle-of-attack flow region, they did not mention effects of Mach number, Reynolds number etc. on the position of the transition point.

Therefore, our interest is how the Mach number and Reynolds number change the flow field over a delta wing with blunt leading edge in supersonic flow region at high angle of attacks. In addition, understanding of the flow field over delta wing with blunt leading edge in supersonic and high angle of attack condition is important in engineering view point as future space plane may fly at such condition in the reentry phase.

The objective of the present research is to experimentally investigate Mach number effect on flow structure over a delta wing with blunt leading edge in supersonic and high angle of attack flow conditions. To achieve this goal, wind tunnel experiments of a $65^{\circ}$ delta wing are performed in supersonic and high angle of attack flow conditions at the JAXA's transonic / supersonic wind tunnel. Oil flow for surface flow visualization, pressure sensitive paint for surface pressure distribution measurement, and Schlieren images for shock wave visualization are used. 


\section{Experimental Setup}

\section{A. Experimental Conditions}

Free-stream Mach number ranges from 0.6 to 3.2. To eliminate Reynolds number effect on the flow over the delta wing, Reynolds number is fixed at $4.65 \times 10^{6}$ by adjusting total of pressure of the incoming flow. Angle of attack is 10 degrees. Figure 2 compares the present test conditions and the flow classification map of Miller and Wood.

\section{B. Wind Tunnel Facility}

Experiments are conducted at JAXA's transonic/supersonic wind tunnel located at Institute of Space and Astronautical Science (ISAS). This wind tunnel has one $60 \times 60\left(\mathrm{~cm}^{2}\right)$ test section for transonic flow region and another $60 \times 60\left(\mathrm{~cm}^{2}\right)$ test section for supersonic flow region. The operation Mach number range of the wind tunnel is $0.3-1.3$ and $1.5-4.0$.

\section{Wind Tunnel Model}

The model is the Euler model ${ }^{10}$, a full-span delta wing mounted on a sting. The sweep angle is 65 degrees and the wing tip is cropped at $85 \%$ span (Fig.3). The wing section is defined by a polynomial function from the $40 \%$ chord to the leading edge with the leading edge radius of $0.7 \%$ chord. The wing section from the $40 \%$ chord is an NACA 64A005 airfoil.

\section{Flow Visualization}

To visualize flow separation and shock waves on the model surface, surface pressure distribution measurement technique using pressure sensitive paint (PSP) and the oil-flow technique are used. Schlieren images are obtained to get information on the flow field above the wing leeward surface.

PSP technique ${ }^{11-14}$ is an optical method that enables measurement of surface pressure distribution over a model basing on oxygen and thermal quenching of luminescent molecules. Here, PSP measurement system based on blue LED and Ruthenium (II) complex is used (Fig.4). The associated image data are processed by using the PSP postprocessing software SMAP ${ }^{15}$. To eliminate temperature dependency of the PSP, surface temperature distribution is measured with temperature sensitive paint (TSP). Detail of the present PSP technique is presented in Ref. 13.

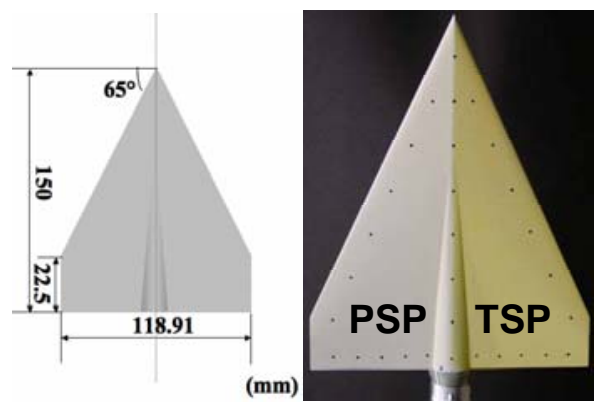

Figure 3. The present test model.

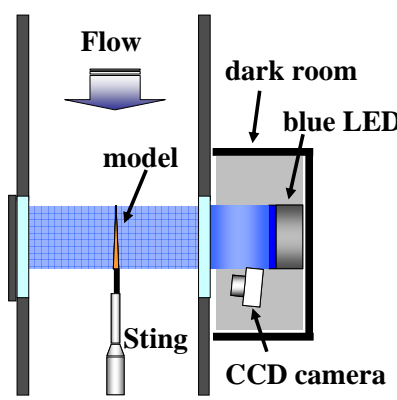

Figure 4. Schematic picture of the surface pressure measurement using PSP.

\section{Results}

Figure 5 is typical oil flow pictures on Schlieren images at Mach number between 1.80 and 3.2. The tests were repeated more than seven times in each flow condition to ensure that the result is qualitatively same. In low supersonic region (free-steam Mach number between 1.2 and 2.0), the flow separated from the apex of the wing and continued to the trailing edge. Oil flow patterns appeared in these flow conditions are characterized by streamwise 
flow inboard, spanwise flow outboard, and no indication of existence of the secondary vortex. This oil flow pattern agrees well with the oil flow pattern of "separation bubble with no shock" in Reference 2. Any strong vortex was not observed in Schlieren images taken from the spanwise direction as shown in Fig. 6. This fact supports that the flow type in this flow condition is separation bubble with no shock.

In high supersonic region (free-stream Mach number between 2.4 and 3.2), while flow separated at the apex of the wing, flow attachment at the leading edge was observed near the trailing edge and the attached flow region expanded toward the apex of the wing as the free-steam Mach number increases. The present experiment showed that flow over a delta wing with blunt leading edge at high angle of attack in supersonic region can be also mixed flow of two different flow types and the position of the transition moves forward as Mach number increases.
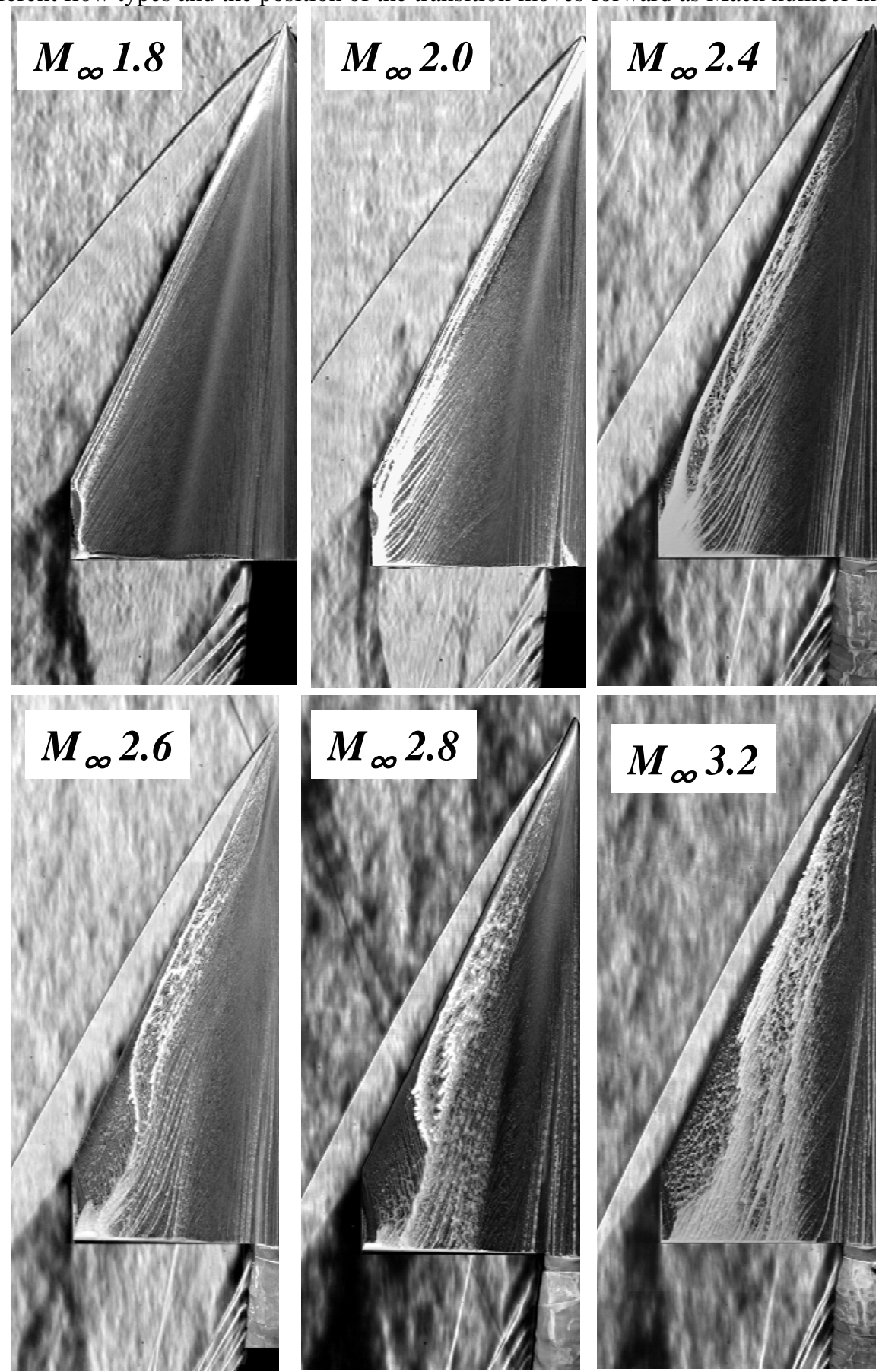

Figure 5. Typical oil flow pictures on Schlieren images. 
Figure 7 is upper surface pressure distribution, oil flow picture, and Schlieren photographs of a typical mixed flow case (free-stream Mach number of 2.6). The oil flow pattern in front region is still that of separation bubble with no shock while the oil flow pattern near the trailing edge agrees with that of "shock-induced separation" in reference 2 . Boundary of the core flow region and separation bubble region are recognizable from the surface pressure distribution as well as the Schlieren photograph. Any strong vortex is not observed on Schlieren images from the side view for all mixed

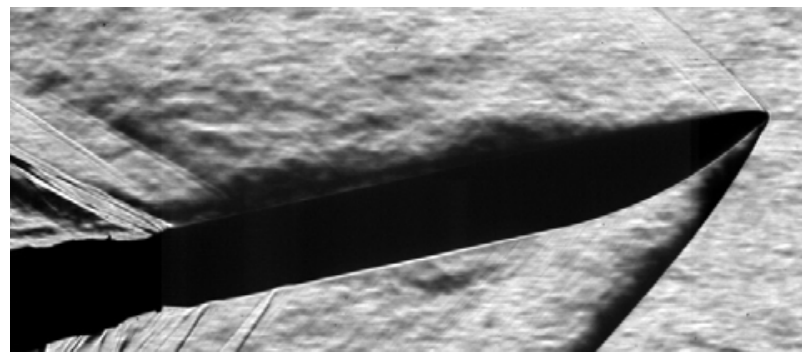

Figure 6. A Schlieren image in spanwise direction at free-stream Mach number of 1.6.

flow cases. This supports the low pressure region is due to bubble instead of vortex.

The mixed flow found by Seshadri and Narayan ${ }^{4}$ for delta wings with sharp leading edge is shock-induced separation with a pair of vortices shed from the wing apex region. Though the mixed flow observed in the current experiments is same as the mixed flow of Seshadri and Narayan in the sense that the flow near the trailing edge is classified as "shock-induced separation", they are different in the front region. This may due to the difference in the leading edge shape.

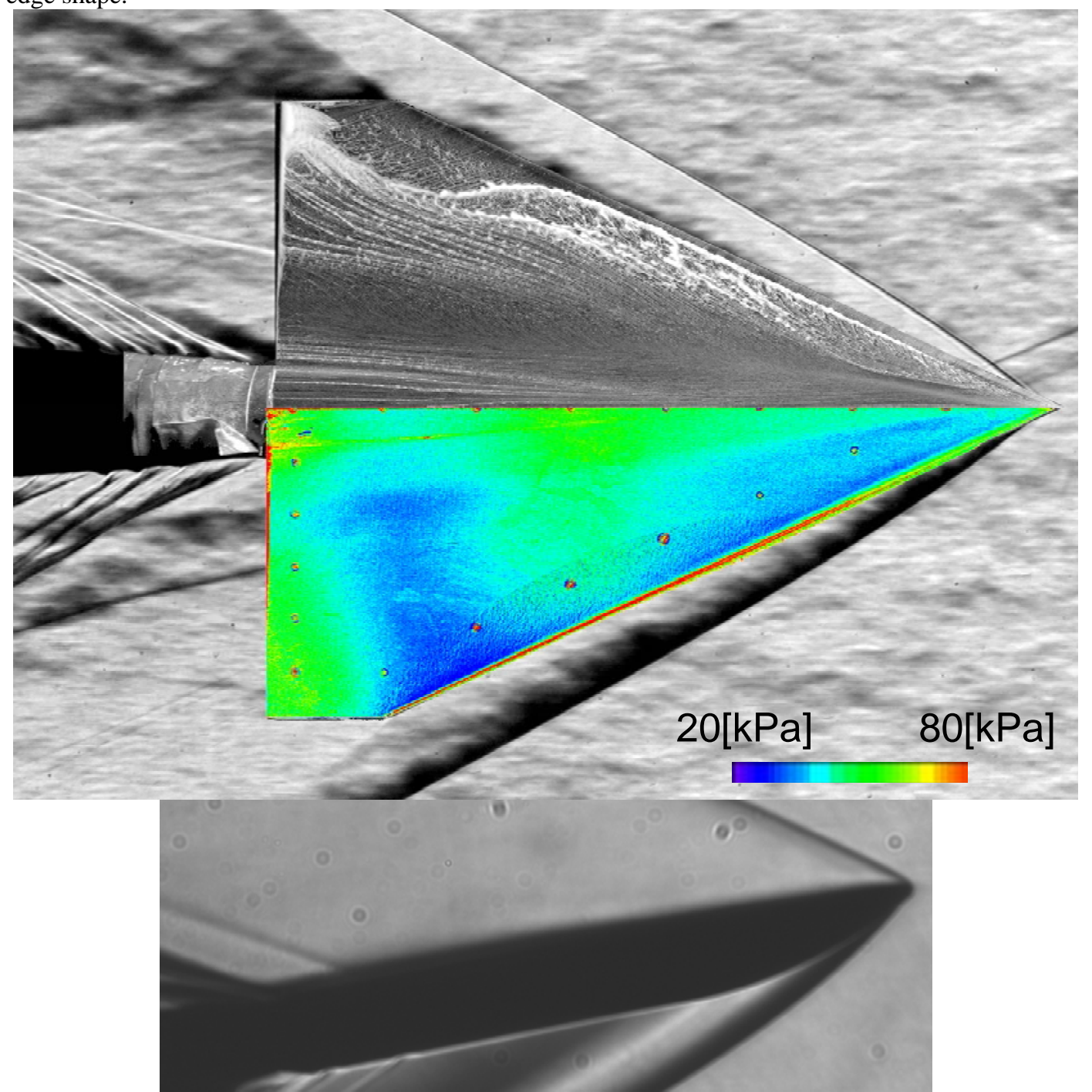

Figure 7. Upper surface pressure distribution (starboard), oil flow pattern (portside), and Schlieren photographs at free-stream Mach number of 2.6 and angle of attack of 10 degrees. 
Figure 8 plots the current experiment conditions on the classification map of Seshadri and Narayan. The flow condition of the mixed flow observed in the current experiments corresponds to the mixed flow region of the classification map of Seshadri and Narayan.

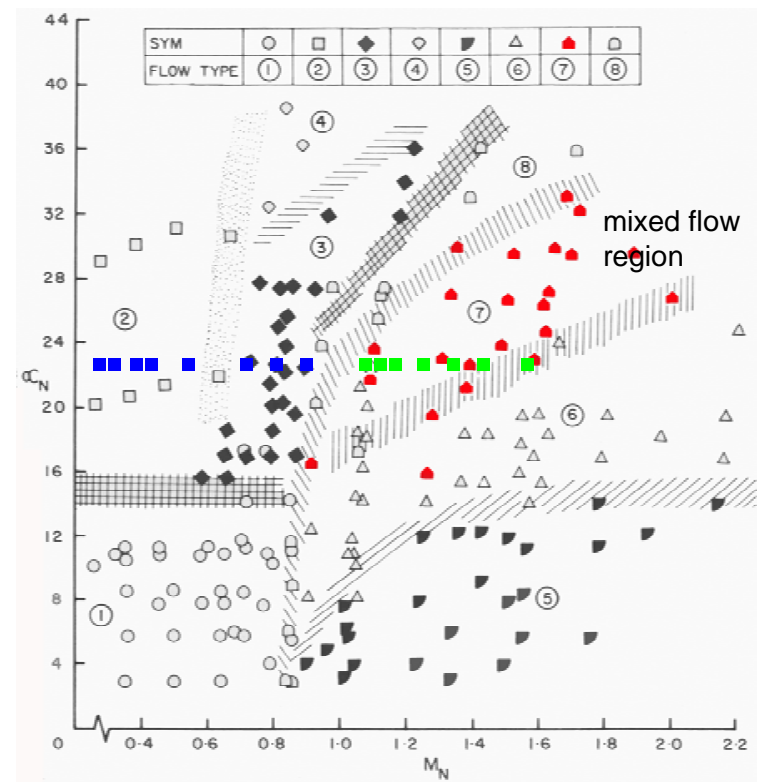

Figure 8. Current experimental conditions compared with the classification map of Seshadri and Narayan ${ }^{4}$. Blue squares are the current experimental conditions where "separation bubble with no shock" was observed. Green squares are the current flow conditions where mixed flow was observed. The red pentagons are flow conditions of Seshadri and Narayan where mixed flow was observed.

Figure 9 plots chordwise positions of boundary of the two different flow types normalized by the root chord length at each Mach number. One of the reasons of scattering is that the position is read from oil flow pictures. This plot confirms that the boundary moves forward as free-stream Mach number increases. This plot also indicates possibility of convergence of the boundary position to $0.40-0.50$ at free-stream Mach number of 3.0 or higher.

\section{Conclusions}

To understand Mach number effect on flow field over a delta wing with blunt leading edge in supersonic and high angle of attack region, wind tunnel experiments of a $65^{\circ}$ delta wing were performed in supersonic and high angle of attack flow conditions at the JAXA's transonic / supersonic wind tunnel. Oil flow for surface flow visualization, pressure sensitive paint for surface pressure distribution measurement, and Schlieren images for shock wave visualization were used.

The present results indicated that a delta wing with

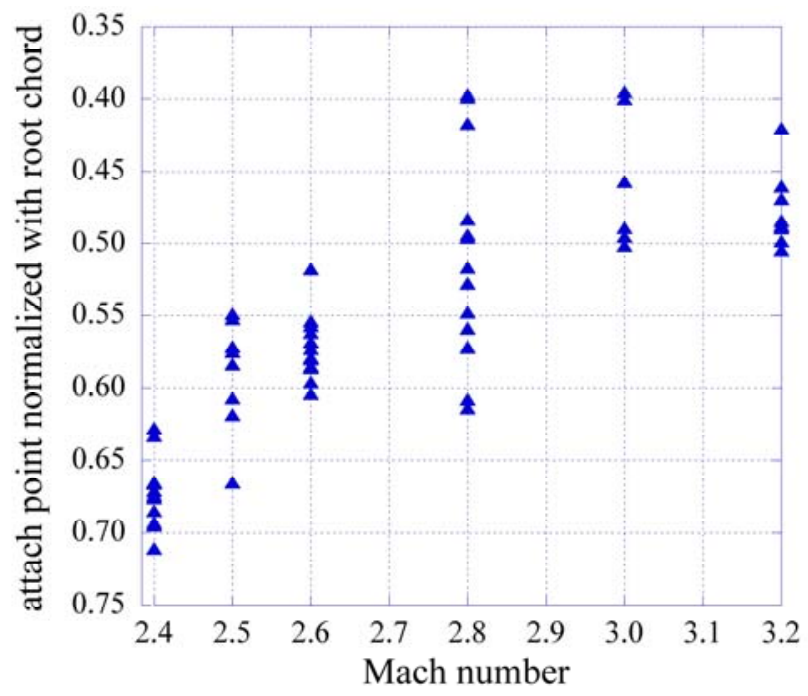

Figure 9. Mach number effect on the location of the boundary between the separated and attached flows at the leading edge.

blunt leading edge can be mixed flow of two different types of flow structure in supersonic and high angle of attack flow region and the location of the boundary of the two types of flow moves toward the apex of the wing as the freestream Mach number increases. 


\section{References}

${ }^{1}$ Stanbrook, A., and Squire, L. C., "Possible Types of Flow at Swept Leading Edges,” Aeronautical Quarterly, Vol.15, No. 2, pp. 72-82, 1964.

${ }^{2}$ Miller, D. S., and Wood, R. M., “Leeside Flows over Delta Wings at Supersonic Speeds,” Journal of Aircraft, Vol. 21, No. 9, pp. 680-686, 1984

${ }^{3}$ Szodruch, J. G., and Peake, D. J., "Leeward Flow over Delta Wings at Supersonic Speeds," NASA-TM, No. 81187, 1980.

${ }^{4}$ Seshadri, S. N., and Narayan, K. Y., "Possible Types of Flow on Lee-Surface of Delta Wings at Supersonic Speeds," Aeronautical Journal, No. 5, pp. 185-199, 1988.

${ }^{5}$ Brodetsky, M. D., Krause, E., Nikiforov, S. B., Pavlov, A. A., Kharitonov, A. M., and Shevchenko, A. M., "Evolution of Vortex Structures on the Leeward Side of a Delta Wing,” Journal of Applied Mechanics and Technical Physics, Vol. 42, No. 2, pp. 242-254, 2001

${ }^{6}$ Imai, G., Fujii, K., and Oyama, A., "Computational Analyses of Supersonic Flows over a Delta Wing at High Angles of Attack," the $24^{\text {th }}$ Congress of the International Council of the Aeronautical Sciences (ICAS), 2006

${ }^{7}$ Luckring, J. M., "Reynolds Number and Leading-Edge Bluntness Effects on a $65^{\circ}$ Delta Wing," AIAA Paper 2002-0419, 2002.

${ }^{8}$ Luckring, J. M., “Transonic Reynolds Number and Leading-Edge Bluntness Effects on a $65^{\circ}$ Delta Wing,” AIAA Paper 2003-0753, 2003.

${ }^{9}$ Luckring, J. M., “Compressibility and Leading-Edge Bluntness Effects for a 65 Delta Wing, AIAA Paper 2004-0765, 2004.

${ }^{10}$ Erickson, G. E., "Wind Tunnel Investigation of the Interaction and Breakdown Characteristics of Slender-Wing Vortices at Subsonic, Transonic, and Supersonic Speeds,” NASA Technical Paper 3114, 1991.

${ }^{11}$ Shimbo, Y. and Asai, K., "Pressure Sensitive Paint Application at Large Production Wind Tunnels," the 22nd Congress of the International Council of the Aeronautical Sciences (ICAS), Paper No. 00.3.3.3, England, 2000.

${ }^{12}$ Liu, T. S., Torgerson, S. D. and Sullivan, J. P., "Rotor Blade Pressure Measurements in a High Speed Axial Compressor using Pressure and Temperature Sensitive Paints,” AIAA Paper 1997-0162, 1997.

${ }^{13}$ Fujimatsu, N. and Nakai, Y. and Fujii, K., "PSP Measurement of the Supersonic Jet Flows Impinging on a Flat plate at Variable Inclined Angles,” ISTS (2002).

${ }^{14}$ Nakai, Y., Fujimatsu, N. and Fujii, K., "Flow Classification of the Under-Expanded Super Sonic Jet Impinging on a Flat Plate," $33^{\text {rd }}$ AIAA Fluid Dynamics Conference \& Exhibit, 2003.

${ }^{15}$ Fujimatsu, N., Fujii, K., Tamura, Y., "Improvement of the Noise Filtering and Image Registration Methods for PSP Experiments,” ASME/FED\&JSME Joint Fluids Conference, Honolulu, 2003.

${ }^{16}$ Ouchi, H., Irikado, T., Fujii, K., and Hayashi, A. K., "PSP Measurements in the Large-Scale Transonic Wind Tunnel and Associated Image Data Processing,” AIAA Paper 2005-1079, 2005. 